\title{
Nucleon axial form factor from generalized parton distributions
}

\author{
Hadi Hashamipour, ${ }^{1, *}$ Muhammad Goharipour, ${ }^{2, \dagger}$ and Siamak S. Gousheh ${ }^{1, \star}$ \\ ${ }^{1}$ Department of Physics, Shahid Beheshti University, General Campus, Evin, Tehran 19839, Iran \\ ${ }^{2}$ School of Particles and Accelerators, Institute for Research in Fundamental Sciences (IPM), PO Box \\ 19568-36681 Tehran, Iran
}

(Received 13 March 2019; revised manuscript received 1 June 2019; published 9 July 2019)

\begin{abstract}
It is well established that the nucleon form factors can be related to generalized parton distributions (GPDs) through sum rules. On the other hand, GPDs can be expressed in terms of parton distribution functions (PDFs) according to the Diehl, Feldmann, Jakob, and Kroll (DFJK) model. In this work, we use this model to calculate polarized GPDs for quarks $\left(\tilde{H}_{q}\right)$ using the available polarized PDFs obtained from the experimental data and then study the axial form factor of nucleon. We determine parameters of the model using standard $\chi^{2}$ analysis of experimental data. It is shown that some parameters should be readjusted, as compared to some previously reported values, to obtain better consistency between the theoretical predictions and experimental data. Moreover, we study in detail the uncertainty of nucleon axial form factor due to various sources.
\end{abstract}

DOI: 10.1103/PhysRevD.100.016001

\section{INTRODUCTION}

Measurement of proton spin asymmetry in the polarized deep inelastic scattering (DIS) experiment performed by European Muon Collaboration in 1987 [1] showed that the total contribution from quark spin to the proton's spin is less than half. Since that time, along with the studies of unpolarized parton distribution functions (PDFs), many experimental, theoretical, and phenomenological investigations have been performed to understand the constituents of the proton's spin and also determine their distributions. In this regard, the determination of polarized PDFs (PPDFs), which describe the structure of a nucleon in a helicity eigenstate, by performing a global analysis of available experimental data has also been of great interest [2-13]. On the other hand, the structure of the nucleon in both the unpolarized and polarized cases can be investigated in more detail using generalized parton distributions (GPDs) [14-28] which comprise important concepts of QCD. Actually, GPDs provide quantitative information on the longitudinal and transverse distribution of partons inside the nucleon and also their intrinsic and orbital

\footnotetext{
*h_hashamipour@sbu.ac.ir

muhammad.goharipour@ipm.ir

*ss-gousheh@sbu.ac.ir
}

Published by the American Physical Society under the terms of the Creative Commons Attribution 4.0 International license. Further distribution of this work must maintain attribution to the author(s) and the published article's title, journal citation, and DOI. Funded by SCOAP ${ }^{3}$. angular momenta. Therefore, studying GPDs can shed light on various aspects of hadron structure.

An accurate knowledge of GPDs is essential for understanding and describing various hard exclusive processes $[14,15,19]$, such as deeply virtual Compton scattering (DVCS), timelike Compton scattering, exclusive meson production by longitudinally polarized photons, and photoproduction of heavy vector mesons [29]. One of the main important properties of GPDs is their mutual relations with PDFs and elastic form factors (FFs). On one hand, GPDs are the off-forward kinematic generalizations of the ordinary PDFs which play a crucial role in inclusive DIS. In other words, PDFs can be recovered from GPDs (in the socalled forward limit) by setting to zero the extra variables in GPDs, such as the transverse momentum between the initial and final protons and skewness parameter. On the other hand, FFs (including the electric and magnetic form factors, or even the form factors associated with the energymomentum tensor), which are other important quantities giving us valuable information on the structure of nucleon, can be obtained from GPDs as well [30-32]. In fact, GPDs give us not only all the information contained in FFs but also other useful information, for example, about the transverse displacement of partons [33]. Consequently, from this point of view, GPDs are a generalization of both PDFs and FFs. It is worth noting that the axial form factors (AFFs) which are fundamental quantities that describe spin content of the nucleon are also intimately related to polarized GPDs (see Sec. II).

Just like PDFs and PPDFs, GPDs are essentially nonperturbative objects, so they cannot be determined directly from the perturbative QCD apart from first Mellin moments 
in special cases in lattice QCD [23,24]. Although early studies of GPDs using various dynamical models of the nucleon structure (see Ref. [28] and references therein) have played an important role in better understanding GPDs and exclusive processes, at the moment, more attention is being paid to determine GPDs from fitting the available experimental data (see Ref. [27] and references therein). Actually, the extraction of GPDs from exclusive processes, for which all particles are detected in the final state, is theoretically well developed. There are valuable experimental data from DVCS on the proton at the HERA collider which cover a wide kinematical region (see Table 4 of Ref. [27]). For the case of DVCS experiments with a fixed proton target, there are also some data on various observables from the HERMES, CLAS, and Hall A collaborations (see Table 5 of Ref. [27]). Nevertheless, DVCS data taking will be continued at CERN by the COMPASS Collaboration [34]. The future measurements in Jefferson Laboratory by CLAS Collaboration [35] with experiments starting at $12 \mathrm{GeV}$ will also provide new information about the valence region. Moreover, one of the main goals of the future Electron-Ion Collider is the measurement of DVCS observables and FFs [36] that makes the extraction of both $H$ and $E$ GPDs possible.

As mentioned, FFs can be written in terms of GPDs, and hence their measurements can give us useful important information about GPDs. In the case of nucleon spin studies, AFFs, which are related to polarized GPDs, can be extracted using various approaches [37-44]. One can find a review of experimental data in Refs. [45,46]. Many lattice QCD calculations of FFs have also been done since the 1980s and have led to considerable results. In recent years, lattice QCD simulations of AFFs have been presented for pion masses in the range $m_{\pi}=0.2-0.6 \mathrm{GeV}$ [47-53]. Very recently, the PACS Collaboration has reported the result of a lattice QCD calculation of nucleon AFF in $2+1$ flavor near the physical pion mass [54]. In addition, neural networks can be applied to extract nucleon AFF from experimental data. In particular, the authors of Ref. [55] have used this tool to analyze the neutrino-deuteron scattering data measured by the Argonne National Laboratory bubble chamber experiment.

In this work, we study the nucleon axial form factor and polarized GPDs, given the fact that they are connected via sum rules. Although there are various models [56-61] and parametrizations [62-64] for GPDs, we use a practical Ansatz suggested by Diehl et al. (DFJK) [30-32], which relates the predetermined (polarized) PDFs as input to (polarized) GPDs. An important advantage of this Ansatz is that it has a few free parameters to be fixed by analyzing experimental data. Considering different scenarios, we determine parameters of the model using standard $\chi^{2}$ analysis of experimental data for nucleon AFFs.

This paper is organized as follows. In Sec. II, the theoretical framework of our study is presented, and we briefly describe the physics related to GPDs and AFFs.
Our method to obtain optimum values for the polarized GPDs of quarks using the available experimental data for nucleon AFFs is also introduced in this section. Section III is devoted to the introduction of the experimental data, which are used in our $\chi^{2}$ analyses. In Sec. IV, we study in detail the nucleon AFF with an emphasis on its dependence on PPDFs according to the DFJK model [30-32] and also the value of scale $\mu^{2}$ associated with the PPDFs. Moreover, we investigate the model uncertainties that are imposed on the nucleon AFF from various sources. In Sec. V, we determine the best values of parameters of the model by performing some $\chi^{2}$ analyses of nucleon AFF data in various scenarios and discuss the results obtained and possible outlooks. Finally, we summarize our results and conclusions in Sec. VI.

\section{THEORETICAL FRAMEWORK}

In this section, we briefly review physical concepts on GPDs and nucleon AFFs and present the theoretical framework we use to obtain optimum values and bounds for polarized GPDs using the available experimental data for nucleon AFFs. As mentioned in the Introduction, GPDs (PDFs) are nonperturbative objects needed for describing hard exclusive (inclusive) electroproduction processes, which are defined as matrix elements of quark and gluon operators at a lightlike separation between two proton states with different (same) momenta. GPDs are also universal objects just like PDFs because they can be defined in the framework of QCD collinear factorization for hard exclusive processes $[65,66]$ such as DVCS and exclusive meson production by longitudinally polarized photons. The importance of GPDs is due to the fact that they contain valuable information on the hadron structure in QCD. Actually, the distributions of quarks and gluons in hadrons in terms of both momentum fractions and the position in the transverse plane can be well described through GPDs.

In the present work, we use the convention of Ji [67] for GPDs, in which $H, E, \tilde{H}$, and $\tilde{E}$ are defined as [15,19]

$$
\begin{aligned}
& \frac{1}{2} \int\left.\frac{d z^{-}}{2 \pi} e^{i x P^{+} z^{-}}\left\langle p^{\prime}\left|\bar{q}\left(-\frac{1}{2} z\right) \gamma^{+} q\left(\frac{1}{2} z\right)\right| p\right\rangle\right|_{\substack{z^{+}=0 . \\
z_{\perp}=0}} \\
&= \frac{1}{2 P^{+}}\left[H^{q}(x, \xi, t) \bar{u}\left(p^{\prime}\right) \gamma^{+} u(p)\right. \\
&\left.+E^{q}(x, \xi, t) \bar{u}\left(p^{\prime}\right) \frac{i \sigma^{+\alpha} \Delta_{\alpha}}{2 m} u(p)\right], \\
&\left.\frac{1}{2} \int \frac{d z^{-}}{2 \pi} e^{i x P^{+} z^{-}}\left\langle p^{\prime}\left|\bar{q}\left(-\frac{1}{2} z\right) \gamma^{+} \gamma_{5} q\left(\frac{1}{2} z\right)\right| p\right\rangle\right|_{\substack{z_{\perp}^{+}=0, z_{\perp}=0}} \\
&=\frac{1}{2 P^{+}}\left[\tilde{H}^{q}(x, \xi, t) \bar{u}\left(p^{\prime}\right) \gamma^{+} \gamma_{5} u(p)\right. \\
&\left.\quad+\tilde{E}^{q}(x, \xi, t) \bar{u}\left(p^{\prime}\right) \frac{\gamma_{5} \Delta^{+}}{2 m} u(p)\right],
\end{aligned}
$$


where $z=\left(z^{+}, z_{\perp}, z^{-}\right)$. As one can readily see from Eq. (1), GPDs have 3 degrees of freedom and then are expressed as functions of three parameter $x, \xi$, and $t$. The first argument is the well-known Bjorken scaling variable (the average momentum fraction) $x=\frac{Q^{2}}{2 p \cdot q}$, with photon virtuality $Q^{2}$. Another longitudinal variable that has a crucial role in GPDs is $\xi=\frac{p^{+}-p^{\prime+}}{p^{+}+p^{\prime+}}$, which is called "skewness." The last argument is $t=\left(p^{\prime}-p\right)^{2}=\Delta^{2}$, i.e., the square of the momentum transferred to the target.

As mentioned, GPDs cannot be calculated from perturbative QCD, but there are some lattice QCD calculations $[23,24]$. A suitable method to extract GPDs is performing a $\chi^{2}$ analysis of experimental data using the factorization theorem. Hard exclusive processes such as DVCS [27] and meson production [32] are the most-used experiments for the extraction of GPDs. As data on hard exclusive processes are much less than inclusive processes, extraction of GPDs from experimental data is not yet feasible with precisions comparable to PDFs. One of the best ways to overcome this problem is using a model for GPDs, but with as few parameters as possible. In this work, we implement the DFJK model [30] for calculating polarized GPDs, which can be expressed in terms of PPDFs and also has few free parameters to be fixed by fitting to nucleon AFF data. We describe the model below.

It is well established now that various nucleon FFs can be related to GPDs through sum rules [32]. For example, the Dirac and Pauli form factors, $F_{1}$ and $F_{2}$, for the proton and neutron can be expressed in the form

$$
\begin{aligned}
& F_{i}^{p}=e_{u} F_{i}^{u}+e_{d} F_{i}^{d}+e_{s} F_{i}^{s}, \\
& F_{i}^{n}=e_{u} F_{i}^{d}+e_{d} F_{i}^{u}+e_{s} F_{i}^{s},
\end{aligned}
$$

where $i=1,2$ and $F_{i}^{q}$ is the contribution from quark flavor $q$ to the nucleon form factor $F_{i}^{A}$, with $A=p, n$. As usual, $e_{q}$ is the electric charge of the quark in units of the positron charge. Now, the flavor form factors $F_{i}^{q}$ can be written in terms of the proton "valence GPDs" $H_{v}$ and $E_{v}$ for unpolarized quarks of flavor $q$ as

$$
\begin{aligned}
& F_{1}^{q}(t)=\int_{0}^{1} d x H_{v}^{q}(x, t), \\
& F_{2}^{q}(t)=\int_{0}^{1} d x E_{v}^{q}(x, t),
\end{aligned}
$$

where valence GPDs $\mathcal{G}_{v}=H_{v}, E_{v}$ for flavor $q$ are expressed in terms of "quark GPDs" $\mathcal{G}$ as

$$
\mathcal{G}_{v}^{q}(x, t)=\mathcal{G}^{q}(x, \xi=0, t)+\mathcal{G}^{q}(-x, \xi=0, t),
$$

with $\mathcal{G}^{q}(-x, \xi=0, t)=-\mathcal{G}^{\bar{q}}(x, \xi=0, t)$. Note that the result, as a consequence of Lorentz invariance, is independent of skewness $\xi$, so one can choose zero skewness GPDs and omit this variable.

As we pointed out, some models for GPDs use ordinary PDFs as input. Considering this fact, PDFs can be defined as

$$
q(x)=\left.\int \frac{d z^{-}}{2 \pi} e^{-i x P^{+} z^{-}}\left\langle p\left|\bar{q}(z) \gamma^{+} q(0)\right| p\right\rangle\right|_{\substack{z^{+}=0, z_{\perp}=0}}
$$

and then recovered from GPDs at the forward limit $(t=0)$. For example, for positive $x$, the GPD $H$ changes to the usual quark and antiquark densities as $H^{q}(x, 0,0)=q(x)$ and $H^{q}(-x, 0,0)=\bar{q}(x)$. According to the DFJK Ansatz [30], which gives $x$ and $t$ dependence of GPDs at zero skewness, the valence GPDs $H_{v}^{q}$, for example, can be related to ordinary valence PDFs as

$$
H_{v}^{q}\left(x, t, \mu^{2}\right)=q_{v}\left(x, \mu^{2}\right) \exp \left[t f_{q}(x)\right],
$$

in which the profile functions $f_{q}(x)$ specify the $x$-dependent width. Actually, this Ansatz assumes an exponential $t$ dependence with a $x$-dependent slope for $H_{v}^{q}$. The profile functions $f_{q}(x)$ can have the simple form shown below, which we shall henceforth call the simple Ansatz:

$$
f_{q}(x)=\alpha^{\prime}(1-x) \log \frac{1}{x} .
$$

This Ansatz, along with a more complex one also given in Ref. [30], was used, for example, in Ref. [31] for the strange Dirac form factor $F_{1}^{s}$. The value of $\alpha^{\prime}$ can be extracted by analyzing the soft hadronic scattering processes like kaonnucleon scattering or photoproduction of the mesons; various analyses have indicated that its values should be close to 1 [30-32]. In our analyses, we consider $\alpha^{\prime}$ to be flavor independent, as suggested by Regge phenomenology [30] and isospin symmetry [32].

In analogy with the Dirac and Pauli FFs, the nucleon axial form factor can be expressed in terms of polarized GPDs as [32]

$$
\begin{aligned}
G_{A}(t)= & \int_{0}^{1} d x\left[\tilde{H}_{v}^{u}(x, t)-\tilde{H}_{v}^{d}(x, t)\right] \\
& +2 \int_{0}^{1} d x\left[\tilde{H}^{\bar{u}}(x, t)-\tilde{H}^{\bar{d}}(x, t)\right] .
\end{aligned}
$$

Note that, for valence polarized GPDs $\tilde{H}_{v}^{q}$, we have

$$
\tilde{H}_{v}^{q}(x, t) \equiv \tilde{H}^{q}(x, \xi=0, t)-\tilde{H}^{q}(-x, \xi=0, t),
$$

with $\tilde{H}^{q}(-x, \xi=0, t)=\tilde{H}^{\bar{q}}(x, \xi=0, t)$. In fact, one can write the quark contribution to AFF generally as an integral of polarized GPDs over Bjorken $x$, 


$$
G_{A}^{q}(t)=\int_{0}^{1} d x \tilde{H}^{q}(x, t),
$$

where $q$ covers here both valence and sea-type contributions of $u p$ and down quarks. To be more precise, Eq. (8) clearly shows that, in contrast to Pauli and Driac FFs, the axial form factor contains also some contributions from the sea quark sector. Although these contributions are not significant compared to those that come from the valence sector, they cannot be neglected. It is worth noting that Eq. (10) is also the intrinsic spin contribution of quark $q$ to the spin of the nucleon.

According to the DFJK model, an Ansatz similar to that shown in Eq. (6) can be also considered for valence polarized GPDs $\tilde{H}_{v}^{q}$, so they can be related to valence polarized PDFs, $\Delta q_{v}(x) \equiv q^{+}(x)-q^{-}(x)$, as

$$
\tilde{H}_{v}^{q}\left(x, t, \mu^{2}\right)=\Delta q_{v}\left(x, \mu^{2}\right) \exp \left[t \tilde{f}_{q}(x)\right],
$$

where $\tilde{f}_{q}(x)$ is the corresponding profile function, which can have again a simple form like Eq. (7) or a complex form with more adjustable parameters. For simplicity, we use the Ansatz in Eq. (11) both for $\tilde{H}_{v}^{q}(x, t)$ and $\tilde{H}^{\bar{q}}(x, t)$ in Eq. (8). In fact, this is an ad hoc Ansatz for $\tilde{H}^{\bar{q}}(x, t)$ of which the physical motivation is not as strong as that of the dominant $\tilde{H}_{v}^{q}(x, t)$.

\section{EXPERIMENTAL DATA}

One of the best ways to investigate the electromagnetic and weak structure of hadrons is using the electroweak probes and then measuring various structure form factors. Actually, the extraction of the electromagnetic nucleon FFs has a long history and remains a popular field of experimental research. An overview and discussion of FF data can be found in Ref. [32]. Although the vector electroweak FFs, which give us valuable information on the spatial distribution of the charge and magnetism, have been explored experimentally to a large extent, our information about the axial form factors is very limited. At the present, there are only two classes of experiments that can be used to determine AFFs: first, (anti)neutrino scattering off protons or nuclei and, second, charged pion electroproduction.

In this section, we introduce the nucleon AFF data that are used in our study. For a clear and thorough review and discussion of AFF data, one can refer to Refs. $[45,46])$. Reference [45] also includes clear explanations about the relevant methods to determine AFF of the nucleon. For the case of (anti)neutrino scattering experiments, we use the data obtained by analyzing the measurements of (quasi) elastic (anti)neutrino scattering off $\mathrm{Ca}, \mathrm{O}$, and $\mathrm{Fe}$ nuclei from MiniBooNE experiments [68]. These data cover a wide range of $-t$ in the interval $0.25<-t<0.9 \mathrm{GeV}^{2}$. As mentioned, the other information on the AFF is obtained from the analysis of charged pion electroproduction off protons, slightly above the pion production threshold. Although such a type of analysis is more complicated, there are more experimental data of this class. In the present work, we use a wide range of charged pion electroproduction data [45,69-78]. In such analyses, the Nambu, Luri, and Shrauner low-energy (NLS) theorem $[79,80]$ is first used for the electric dipole amplitude $E_{0+}^{(-)}$at production threshold. Note that the NLS theorem is valid for soft pions, namely, pions that have vanishing 4-momentum. Then model dependent corrections, labeled as soft pion, Furlan Paver Verzegnassi, Dombey Read, and Benfatto Nicolo Rossi, are used to connect the low-energy theorem to the data, in other words, to the realistic case with a finite pion mass (see [45] and references therein).

In most cases, the AFF data are presented as a simple parametrization [45]. The commonly used parametrization for AFFs is the so-called dipole Ansatz, for its $t$ dependence, which is as follows,

$$
G_{A}^{\text {dipole }}(-t)=\frac{g_{A}}{1+\frac{-t}{M_{A}^{2}}},
$$

where the value of axial mass $M_{A}$ varies between 1.03 and $1.07 \mathrm{GeV}$ depending on the method which is used for analyzing experimental data $[45,46]$. The value of $G_{A}$ at $t=0$, the axial charge $g_{A}$, is precisely determined from $\beta$-decay experiments. As can be seen, Eq. (12) has only a single free parameter, $M_{A}$, which should be fixed by fitting the experimental data. It should be also noted that, in the Breit frame and for small momenta, such $-t$ dependence of AFFs leads to an exponential decrease for the axial charge distribution [55]. However, from a theoretical point of view, it has been indicated that this Ansatz is not a good choice; e.g., see Refs. [81,82]. For example, a recent analysis of $G_{A}$ [83], using conformal mapping or $\mathrm{z}$ expansion, shows that the dipole Ansatz systematically underestimates the uncertainty of AFFs. Therefore, in this work, we do not implement the dipole Ansatz and use the experimental data points directly.

Another important point which should be noted is that the experimental data of Refs. [68-78] for AFFs have been presented as the ratio to $G_{A}$ at $t=0$, i.e., $G_{A}(-t) / G_{A}(0)$. Hence, one can use two approaches for analyzing these data: 1) using the original data as ratios and 2) using data as $G_{A}(-t)$. In the next section, we first use both of them and compare their results and then continue our investigations with just $G_{A}(-t)$ data. Note that for extracting $G_{A}(-t)$ data from original $G_{A}(-t) / G_{A}(0)$ data we need the value of $G_{A}(0)$ (axial charge $g_{A}$ ). Although more accurate results for $g_{A}$ can be extracted from recent measurements of the nucleon lifetime [84], we use the latest value from particle data group [85], i.e., $g_{A}=1.2723 \pm 0.0023$.

As a last point, note that the total number of data points from Refs. [68-78] that we can use in our study is 84 . However, comparing data points from various experiments, one finds that some of points have the same $-t$. 


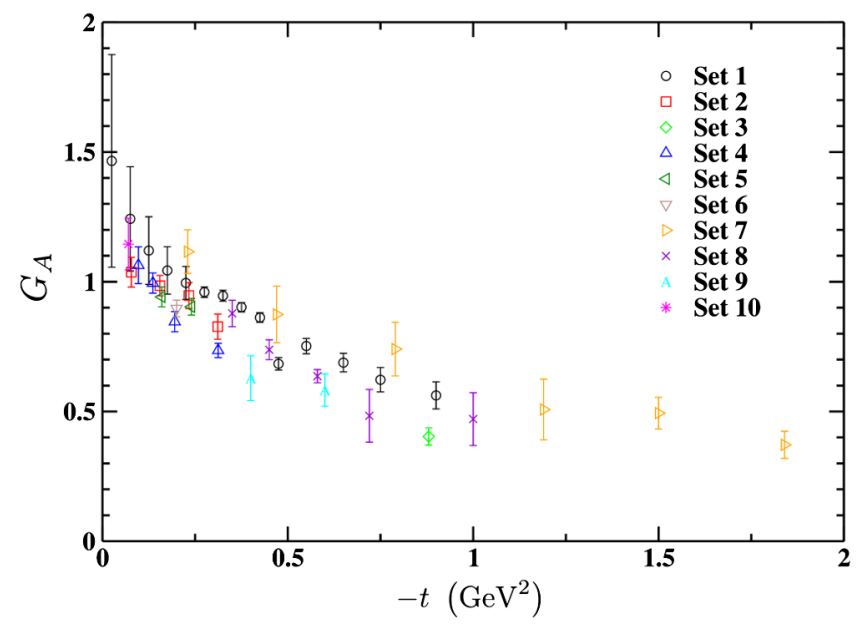

FIG. 1. A comparison between all datasets forming "reduced data" taken from Refs. [68-77]. See the text for further details.

Therefore, another way to analyze these data is removing those with the same value of $-t$ and retaining the most accurate ones. If we do this, 40 data points will remain, and we refer to them as "reduced data" in the following. All datasets forming reduced data have been plotted in Fig. 1. As can be seen, although these datasets have a similar trend in $-t$, there are many inconsistent data. Moreover, these inconsistencies are such that one cannot put them into certain classes. Our investigations indicate that these inconsistencies are not due to the normalization. Half of the sets have less than three data points, and this makes a separate fit of each set impractical. Hence, we use all the data shown in Fig. 1 for our analysis.

\section{STUDY OF NUCLEON AXIAL FORM FACTOR}

In the previous sections, we presented the theoretical framework and experimental information related to the nucleon axial form factor $G_{A}$. In this section, we study $G_{A}$ in detail with emphasis on its dependence on PPDFs according to the Ansatz of Eq. (11) and also the value of scale $\mu$ at which PPDFs are chosen. Moreover, we investigate the model uncertainties that are imposed upon the nucleon AFFs due to the PPDFs uncertainties and also the variation of $\alpha^{\prime}$ in profile functions $f(x)$.

\section{A. Dependence of $G_{A}$ on the PPDFs}

As can be seen from Eq. (8), the nucleon axial form factor can be related to PPDFs through its dependence on polarized GPDs and the relationship between polarized GPDs and PPDFs. It is natural to expect that using different sets of PPDFs to perform calculations should not change the behavior and magnitude of the resulting $G_{A}$; otherwise, the model can be considered not flexible enough or inconsistent. Consequently, in this section, we choose the simple Ansatz given by Eq. (7) and calculate $G_{A}$ using different sets of PPDFs and compare the results to see if

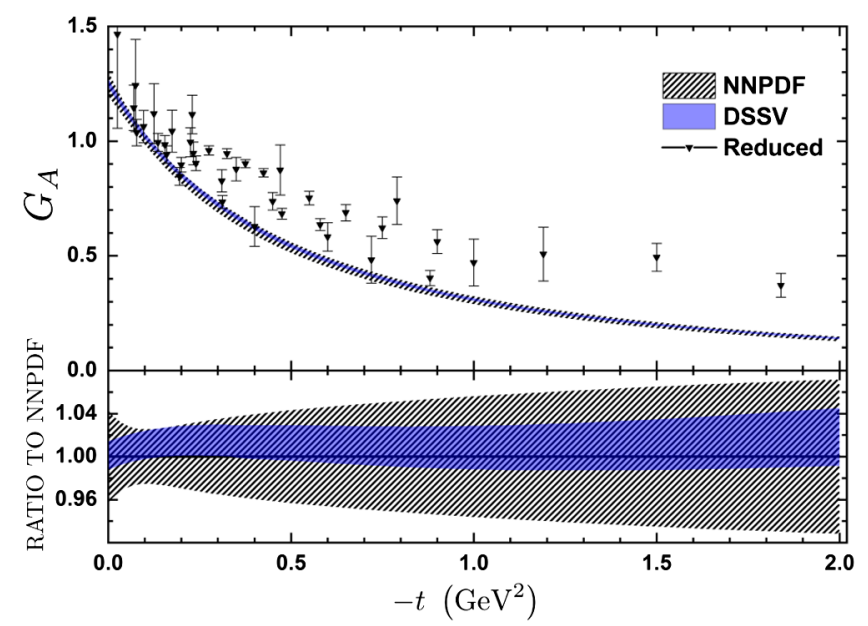

FIG. 2. The theoretical results obtained for nucleon AFFs, $G_{A}$, as a function of $-t$ using the simple Ansatz of Eq. (7) with nextto-leading-order PPDF sets DSSV08 [2] (filled band) and NNPDFPOL1.1 [6] (hachured band) taken at $\mu=2 \mathrm{GeV}$; the value of $\alpha^{\prime}$ is set to $0.95 \mathrm{GeV}^{-2}$. The data points labeled as "reduced" are related to various experiments selected in a procedure explained in Sec. III.

such dependence is present. We will show below that such dependence is not present and hence the model can be used to describe the data.

Since the evaluation of the uncertainty in $G_{A}$ due to the PPDF uncertainties is also of interest, we choose two next-to-leading-order (NLO) PPDF sets DSSV08 [2] and NNPDFPOL1.1 [6], which provide error PPDF sets in addition to their central fit results. ${ }^{1}$ An important advantage of these sets is that one can calculate the uncertainties in any quantity related to PPDFs more easily [87,88]. Figure 2 shows the results obtained for nucleon axial form factor $G_{A}$ as a function of $-t$ in which the hachured and filled bands correspond to the NNPDFPOL1.1 and DSSV08 PPDFs, respectively. To investigate in more detail the differences between the predictions in various regions of $-t$, their ratios to the NNPDFPOL1.1 prediction have also been plotted in the bottom panel of Fig. 2. Moreover, the experimental data from various experiments, which as explained in the previous section are referred to as reduced, have been shown for comparison. Both DSSV08 and NNPDFPOL1.1 PPDFs have been taken at $\mu=2 \mathrm{GeV}$ as suggested in Refs. [30-32]. Note also that the value of $\alpha^{\prime}$ in Eqs. (7) and (11) has been set to $\alpha^{\prime}=0.95 \mathrm{GeV}^{-2}$, which is in conformity with that which has been used in the study performed in Ref. [31] on the strange Dirac form factor $F_{1}^{s}$.

According to the results obtained, one can conclude that if the Ansatz of Eq. (11) is used for calculating $G_{A}$ the final results will not be remarkably sensitive to the choice of

\footnotetext{
${ }^{1}$ The NNPDFPOL1.1 PPDFs are available through the LHAPDF package [86].
} 


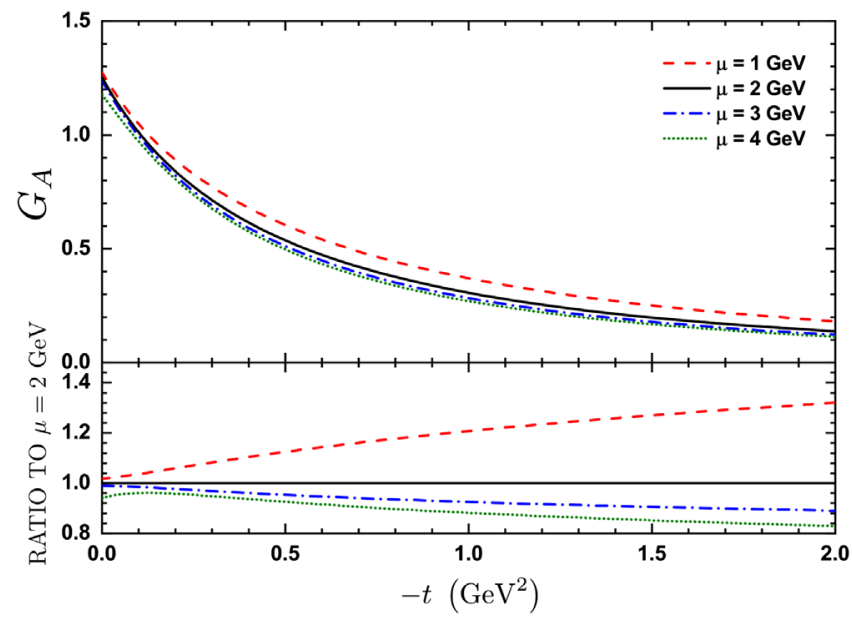

FIG. 3. The dependence of $G_{A}$ on the value of scale $\mu$ in which PPDFs are chosen. The calculations for the model have been performed using the simple Ansatz in Eq. (11) $\left(\alpha^{\prime}=0.95 \mathrm{GeV}^{-2}\right)$, NLO PPDFs of NNPDFPOL1.1 [6] taken at $\mu=1$ (dashed), 2 (solid), 3 (dotted-dashed), and 4 (dotted) $\mathrm{GeV}$. The ratios of the predictions to the corresponding result of $\mu=2 \mathrm{GeV}$ are shown in the bottom panel.

PPDFs set. To be more precise, according to the bottom panel of Fig. 2, the difference between the results obtained for $G_{A}$ using the DSSV08 and NNPDFPOL1.1 PPDFs is almost less than $2 \%$ in full range of $-t$, though the amounts of their uncertainties are somewhat different. However, Fig. 2 clearly shows that the model fails to represent the data. As we shall show below, we can obtain an acceptable fit with a readjustment of parameters $\alpha^{\prime}$ and $\mu$.

\section{B. Dependence on the scale $\mu$ of PPDFs}

Although the results presented in the previous subsection for the nucleon AFFs, $G_{A}$, using the simple Ansatz of Eq. (7) follow roughly the experimental data, the question to be answered is to what extent the results will change if we take PPDFs at scales other than $\mu=2 \mathrm{GeV}$. In Refs. [30], the authors explained that the choice of scale should be a compromise between being large enough for PPDFs, $\Delta q_{v}(x)$, to be rather directly fixed by data and small enough to make contact with soft physics like conventional Regge phenomenology. However, since the recent analysis of PPDFs performed by the NNPDF Collaboration, namely, NNPDFPOL1.1 [6], included a wide range of the available experimental data which covers the lower values of $\mu$ down to $\mu=1 \mathrm{GeV}$, it is also of interest to study the impact of taking PPDFs at a scale different from $\mu=2 \mathrm{GeV}$ on the theoretical predictions of $G_{A}$ using the simple Ansatz of Eq. (7). That is, one of the goals of this paper is to find a fixed $\mu$ at which the Ansatz given in Eq. (11) produces the best fit to the data for $G_{A}$. However, it should be also noted that the scale dependence of GPDs is known. For the case of zero skewness GPDs, this scale dependence is given by the well-known DGLAP evolution equations. To be more precise, GPDs depend on the factorization scale $\mu$ at which the partons are resolved, just like the usual quark densities. We refer the interested reader to Ref. [30] for further details.

To evaluate the dependence of $G_{A}$ on the value of scale $\mu$ in which PPDFs are chosen, we repeat here the calculations performed in the previous subsection using the NNPDFPOL1.1 PPDFs but at different values of scale $\mu$. As mentioned above, the functional dependence of the PPDFs, extracted from NNPDFPOL1.1, on $\mu$ are in accordance with the DGLAP evolution equation. The results obtained have been shown in Fig. 3, in which the dashed, solid, dotteddashed, and dotted curves correspond to NNPDFPOL1.1 PPDFs taken at $\mu=1,2,3$, and $4 \mathrm{GeV}$, respectively. To make a better comparison, in the bottom panel, we have also plotted the ratios of the predictions to the corresponding result obtained using NNPDFPOL1.1 PPDFs taken at $\mu=2 \mathrm{GeV}$ as a reference. As can be clearly seen, by decreasing the value of $\mu$ in which PPDFs are chosen, $G_{A}$ increases especially for larger values of $-t$, so the difference between the results of $\mu=1$ and $\mu=2 \mathrm{GeV}$ reaches even to $30 \%$ at $-t=2 \mathrm{GeV}^{2}$. On the other hand, as the value of $\mu$ increases, $G_{A}$ decreases, but with a smaller rate than before, such that the difference between the results of $\mu=2$ and $\mu=4 \mathrm{GeV}$ reaches only $20 \%$ at $-t=2 \mathrm{GeV}^{2}$. Comparing the results of Figs. 2 and 3, one can conclude that taking PPDFs at a lower scale $\mu$ can lead to a better description of the experimental data and lessen the relatively large discrepancy observed in Fig. 2 between the predictions of the model and experimental data.

\section{Model uncertainties}

After studying the dependence of the nucleon axial form factor $G_{A}$ on the PPDFs and also the value of scale $\mu$ at which they are chosen, now we investigate the amount of uncertainties imposed on predictions of the model for $G_{A}$ due to various sources and compare them with each other. According to the sum rule given in Eq. (8), the model uncertainties in $G_{A}$ can arise from the PPDFs uncertainties, the uncertainty of the scale $\mu$ in which PPDFs are chosen, and the uncertainty of $\alpha^{\prime}$ in the profile function $f(x)$. We have studied the first two in Figs. 2 and 3, respectively, and here investigate the uncertainties which arise from the $\alpha^{\prime}$ variation. For this purpose, we repeat the calculations performed in Fig. 2 using NNPDFPOL1.1 PPDFs [6] taken at $\mu=2 \mathrm{GeV}$, but this time vary $\alpha^{\prime}$ in the range $0.85 \mathrm{GeV}^{-2}<\alpha^{\prime}<1.15 \mathrm{GeV}^{-2}$.

Figure 4 shows a comparison between the model uncertainties in $G_{A}$ due to the PPDFs uncertainties (filled band) and $\alpha^{\prime}$ variations (hachured band) in aforementioned range. The bottom panel shows the relative uncertainties obtained by dividing the upper and lower bands of each prediction by its central value. As can be seen, the uncertainty arising from the $\alpha^{\prime}$ variations is remarkably 


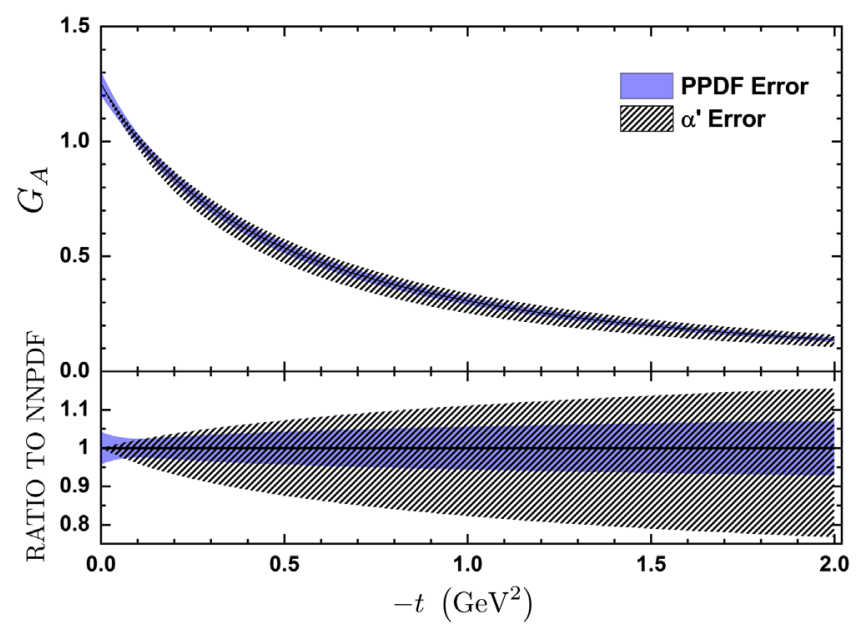

FIG. 4. A comparison between the model uncertainties in $G_{A}$ due to the PPDFs uncertainties (filled band) and $\alpha^{\prime}$ variations (hachured band) in the range $0.85 \mathrm{GeV}^{-2}<\alpha^{\prime}<1.15 \mathrm{GeV}^{-2}$. The theoretical calculations have been performed using the simple Ansatz of Eq. (7) with NLO PPDFs of NNPDFPOL1.1 [6] taken at $\mu=2 \mathrm{GeV}$. The bottom panel shows the relative uncertainties.

dominant compared to the PPDFs uncertainty, except for very small values of $-t$ in which the PPDFs uncertainty becomes dominant. Note also that the uncertainty due to $\alpha^{\prime}$ variations is asymmetric, while the PPDFs uncertainty is symmetric.

\section{V. $\chi^{2}$ ANALYSIS OF THE EXPERIMENTAL DATA}

In the previous section, we found that the nucleon axial form factor $G_{A}$ is not sensitive to the set of PPDFs chosen if an Ansatz like Eq. (11) is used for connecting $G_{A}$ to PPDFs via GPDs. However, according to the results obtained, any change in the scale $\mu$ in which the PPDFs are taken and also the value of $\alpha^{\prime}$ in profile function $f(x)$ can lead to different results for $G_{A}$. For this reason, in this section, we compute the best values for parameters of the model, i.e., $\mu$ and $\alpha^{\prime}$, by performing $\chi^{2}$ analyses of the available experimental data. The optimization is done by the CERN program MINUIT [89].

\section{A. Simple Ansatz}

To determine the best values of $\mu$ and $\alpha^{\prime}$ which are consistent with the experimental data of the nucleon axial form factor, various scenarios can be considered. In a first step, we perform a $\chi^{2}$ analysis of all data for the ratio $G_{A}(-t) / G_{A}(0)$ from various experiments introduced in Sec. III. For the theoretical calculations, we consider the simple Ansatz of Eq. (7) with the NNPDFPOL1.1 [6] as input PPDFs. Note that the theoretical calculation of quantity $G_{A}(-t) / G_{A}(0)$ is not sensitive to the value of scale $\mu$ in which PPDFs are chosen, since it is performed according to Eq. (8) and hence both the numerator and dominator include PPDFs similarly. We have examined various values of $\mu$ and found that the results for the value of $\alpha^{\prime}$ determined from the fit do not change up to four decimal places.

With 84 data points and 1 free parameter, the value of $\chi^{2}$ divided by the number of degrees of freedom is equal to $\chi^{2} /$ d.o.f. $=4.237$. As discussed in Sec. III, the reason for the large $\chi^{2} /$ d.o.f. obtained from the fit is that there are many inconsistent data. Even when we exclude some of datasets from the analysis, the value of $\chi^{2} /$ d.o.f. does not change drastically. Overall, since datasets cannot be categorized and inconsistencies do not come from just a few points, we have opted to make an overall fit to all data instead of making separate fits. The value of $\alpha^{\prime}$ extracted from the fit is

$$
\alpha^{\prime}=2.754 \pm 0.0058 \mathrm{GeV}^{-2},
$$

which is larger than the result obtained in Ref. [31] (about $\left.1 \mathrm{GeV}^{-2}\right)$. Using the reduced dataset for $G_{A}(-t) / G_{A}(0)$, which includes the most precise point among the data points with the same $-t$ (40 data points), the value of $\alpha^{\prime}$ is changed to $\alpha^{\prime}=2.476 \pm 0.0064 \mathrm{GeV}^{-2}$. However, the value of $\chi^{2} /$ d.o.f. increases to 5.129 , since more than 40 data point with larger uncertainties have been removed from the analysis.

Since, as mentioned before, the quantity $G_{A}(-t) / G_{A}(0)$ cannot put any constraint on the value of scale $\mu$ at which PPDFs are chosen, the above values obtained for $\alpha^{\prime}$ are not very reliable. Actually, according to the results obtained in the previous section, we know that the change in $\mu$ can change the result of $G_{A}$ and subsequently the best value of $\alpha^{\prime}$. Consequently, it is more reliable to extract the value of $\mu$ by performing a $\chi^{2}$ analysis of $G_{A}$ data. For this purpose, we consider the reduced data for $G_{A}$ that have been obtained from the original measurements of $G_{A}(-t) / G_{A}(0)$, using $G_{A}(0)=1.2723 \pm 0.0023$ [85].

Since the quantity $G_{A}(-t)$ is sensitive to the value of both $\mu$ and $\alpha^{\prime}$, we can determine their optimal values simultaneously. For this purpose, we can follow two procedures: 1) performing several $\chi^{2}$ analyses by choosing different values for $\mu$ as a fixed parameter and then minimizing $\chi^{2}$ with respect to $\alpha^{\prime}$ and then plotting the $\chi^{2}$ as a function of $\mu$ to find the point at which $\chi^{2}$ is an absolute minimum and its corresponding $\alpha^{\prime}$ (we call this procedure "minimum tracing") and 2) taking both $\mu$ and $\alpha^{\prime}$ as free parameters and minimizing $\chi^{2}$ with respect to both simultaneously. By following these two procedures, we can also find if there is a correlation between $\mu$ and $\alpha^{\prime}$.

Figure 5 shows the results obtained for the minimum tracing of $\chi^{2} /$ d.o.f. values of the reduced $G_{A}$ data as a function of $\mu$ in which PPDFs are chosen in the calculation of the nucleon AFFs (8). As can be seen, for the very small values of $\mu$, the $\chi^{2}$ arises rapidly, while for the values greater than 1 , it increases slowly. Note that the 


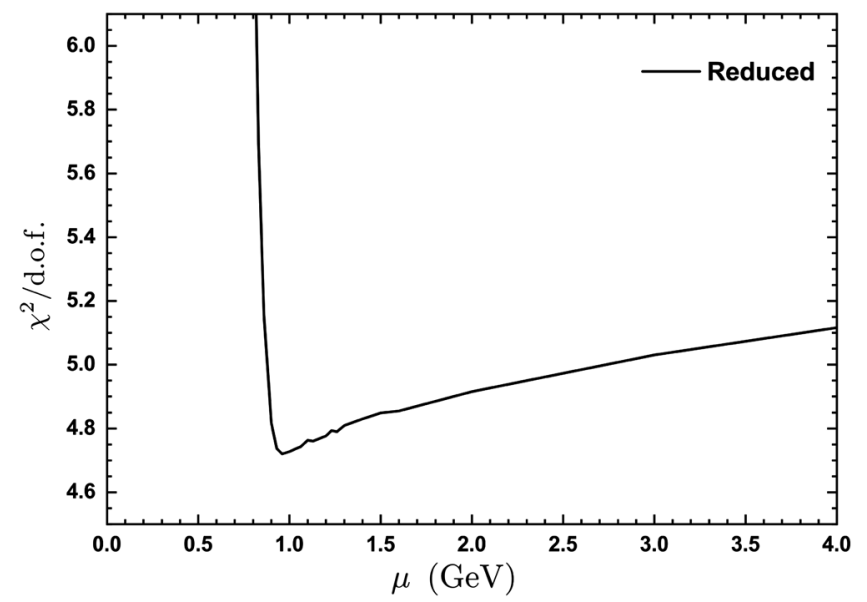

FIG. 5. The minimum tracing of $\chi^{2} /$ d.o.f., shows the minimum of $\chi^{2} /$ d.o.f. as a function of $\mu$ in which PPDFs are chosen in calculation of the nucleon AFFs, Eq. (8), for the analysis of reduced $G_{A}$ data utilizing the simple Ansatz for the profile function.

minimum occurs at about $\mu=0.96 \mathrm{GeV}$, which is smaller than the value considered by the authors of Ref. [31] $(\mu=2 \mathrm{GeV})$. Moreover, the value of $\alpha^{\prime}$ corresponding to this minimum is now

$$
\alpha^{\prime}=0.59 \pm 0.0014 \mathrm{GeV}^{-2},
$$

which is also smaller than the result obtained in Ref. [31].

As mentioned earlier, another method is to find the best values of $\mu$ and $\alpha^{\prime}$ from the $\chi^{2}$ analysis of reduced $G_{A}$ data simultaneously. By performing such an analysis using MINUIT [89], the results

$$
\begin{aligned}
\alpha^{\prime} & =0.59 \pm 0.0022 \mathrm{GeV}^{-2} \\
\mu & =0.962 \pm 0.0098 \mathrm{GeV}
\end{aligned}
$$

are obtained at are, as expected, the same as those of the minimum tracing method. If we use all of the $G_{A}$ data rather than its reduced set, these values are changed to

$$
\begin{aligned}
\alpha^{\prime} & =0.65 \pm 0.0014 \mathrm{GeV}^{-2}, \\
\mu & =0.987 \pm 0.11 \mathrm{GeV}
\end{aligned}
$$

The sensitivity of $\chi^{2}$ to the value of $\mu$ and $\alpha^{\prime}$ can be also studied in detail by plotting a contour plot which includes appropriate ranges of two parameters $\mu$ and $\alpha^{\prime}$. Figure 6 shows the contour plot of $\chi^{2} /$ d.o.f. as a function of free parameters $\mu$ and $\alpha^{\prime}$. As can be seen from this figure, a steep rise in $\chi^{2} /$ d.o.f. occurs, if we increase or decrease $\alpha^{\prime}$ more than 0.1 away from the best value. This fact means that the value of $\chi^{2} /$ d.o.f. is very sensitive to $\alpha^{\prime}$ and that the reduced $G_{A}$ data can put a good constraint on $\alpha^{\prime}$. This is consistent with the small uncertainties for $\alpha^{\prime}$ given in Eqs. (7) and (16).

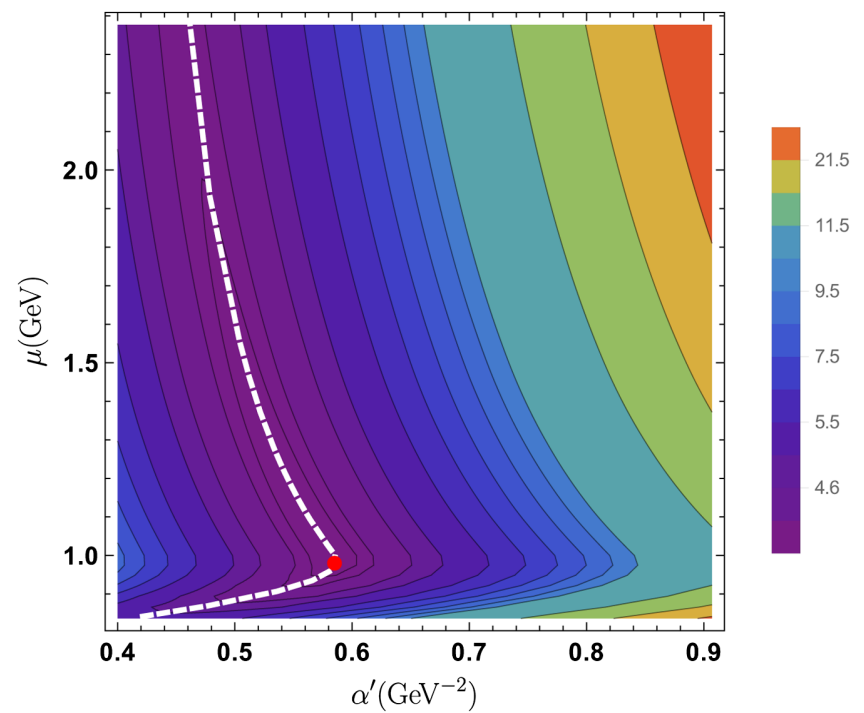

FIG. 6. Contour plot of $\chi^{2} /$ d.o.f. as a function of free parameters $\mu$ and $\alpha^{\prime}$ for the simple Ansatz. The value of $\chi^{2} /$ d.o.f. is shown by colors (see the bar legend on the right). The best value of $\chi^{2} /$ d.o.f. is shown as a red dot, and the dashed line shows the path taken in Fig. 5 for the minimum tracing procedure (see the text for more details).

The situation is somewhat different for parameter $\mu$. Actually, a steep rise in the $\chi^{2} /$ d.o.f. occurs for $\mu>2$ or $\mu<0.9$, but for $0.9 \lesssim \mu \lesssim 2$, one can see that $\chi^{2} /$ d.o.f. do not change as much. The minimum value of $\chi^{2} /$ d.o.f. has been shown as a red dot in the figure and occurs in $\mu$ and $\alpha^{\prime}$ values shown in Eq. (15). The dashed line shows the path in the $\alpha^{\prime}-\mu$ plane taken in Fig. 5 for finding the minimum value of $\chi^{2} /$ d.o.f. using our minimum tracing procedure. In other words, the dashed line in Fig. 6 shows the correspondence between the two procedures for finding the minimum of $\chi^{2} /$ d.o.f. explained earlier in this section; each point on this curve shows the pair $\alpha^{\prime}$ and $\mu$ for which Fig. 5 has a corresponding $\mu$ and $\chi^{2} /$ d.o.f. pair.

Figure 7 shows a comparison between the theoretical predictions for the nucleon axial form factor $G_{A}$ using the values obtained for $\alpha^{\prime}$ and $\mu$ [Eq. (15)] from the analysis of reduced $G_{A}$ data (filled band) and the results obtained using default values $\alpha^{\prime}=0.95 \mathrm{GeV}^{-2}$ and $\mu=2 \mathrm{GeV}$ (hachured band). The data points are those of the reduced set. As can be seen, the theoretical prediction is now in more consistent with the experimental data.

In hard exclusive pion electroproduction in which $\tilde{H}$ plays an important role, usually a profile function with a factor $\alpha^{\prime} \log 1 / x+B$ is used rather than the simple Ansatz of Eq. (7) (see, for example, Ref. [90]). Therefore, it is also of interest to calculate the results for the following profile function with the same power of $(1-x)$ in both terms,

$$
f_{q}(x)=\left(\alpha^{\prime} \log \frac{1}{x}+B_{q}\right)(1-x) .
$$




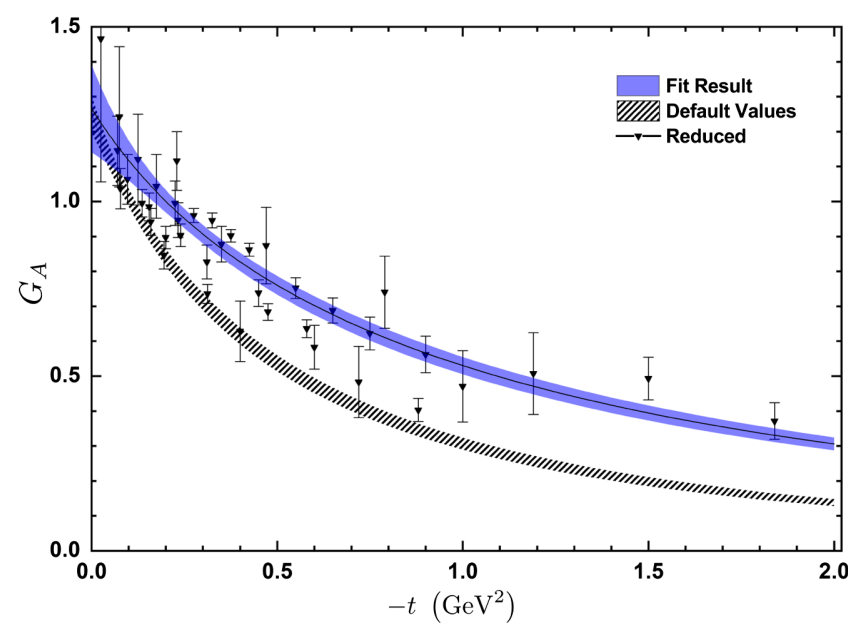

FIG. 7. A comparison between the reduced experimental data and theoretical predictions for $G_{A}$ using the values obtained for $\alpha^{\prime}$ and $\mu$ [Eq. (15)] from the fit (filled band) and the results obtained using default values of Ref. [31], $\alpha^{\prime}=0.95 \mathrm{GeV}^{-2}$ and $\mu=$ $2 \mathrm{GeV}$ (hachured band).

The results of the fit are shown below:

$$
\begin{aligned}
\alpha^{\prime} & =0.683 \pm 0.09 \mathrm{GeV}^{-2} \quad \mu=0.964 \pm 0.11 \mathrm{GeV} \\
B_{v} & =-0.179 \pm 0.19 \mathrm{GeV}^{-2} \quad \chi^{2} / \text { d.o.f. }=4.95
\end{aligned}
$$

The use of this profile function leads to a decrease in the value of $\chi^{2}$ relative to when we use the Ansatz of Eq. (7). However, the value of $\chi^{2} /$ d.o.f. is increased slightly because of the increase in the number of adjustable parameters. We should mention that, although both of the Ansätze used in this subsection give an acceptable fit to the data, they both yield $\alpha^{\prime} \approx 0.7 \mathrm{GeV}^{-2}$, which is lower than the value $\alpha^{\prime} \approx 1 \mathrm{GeV}^{-2}$ obtained in Refs. [30,31]. In the next subsection, we use more flexible Ansätze, which eliminate this discrepancy.

\section{B. Complex Ansatz}

Although Fig. 7 clearly shows that using the simple Ansatz of Eq. (7) can lead to an acceptable fit of the nucleon axial form factor $G_{A}$ data, it is also of interest to investigate the effect of considering a more flexible profile function. In Ref. [30], the authors indicated that low- and high- $x$ behavior of profile function $f(x)$ and also the intermediate- $x$ region can be well characterized by the forms

$f_{q}(x)=\alpha^{\prime}(1-x)^{2} \log \frac{1}{x}+B_{q}(1-x)^{2}+A_{q} x(1-x)$,

and

$f_{q}(x)=\alpha^{\prime}(1-x)^{3} \log \frac{1}{x}+B_{q}(1-x)^{3}+A_{q} x(1-x)^{2}$.
As mentioned at the end of last subsection, both simple Ansätze used there yield values for $\alpha^{\prime}$ which are too low. In fact, one cannot expect both the small- and large- $x$ behavior of GPDs to be controlled only by a single parameter $\left(\alpha^{\prime}\right)$. Moreover, the intermediate- $x$ region should interpolate smoothly between the two limits. Hence, we expect that adding a few extra terms with an appropriate form which include adjustable parameters will improve the results. Actually, the factors of $(1-x)$ in profile function $f_{q}(x)$ ensure that the transverse size of the proton in the limit of $x \rightarrow 1$ remains finite and also decrease the role of $\alpha^{\prime}$ in that limit. We refer the interested reader to Ref. [30] for more explanation.

In this section, we examine these profile functions to see whether any improvement in the theoretical predictions and the fit can be achieved. Note that for the calculation of $G_{A}$ according to Eq. (8), one, in principle, needs to consider profile function (18) [or Eq. (19)] for each flavor $u_{v}, d_{v}, \bar{u}$, and $\bar{d}$. In this way, there are eight more free parameters that should be determined from the analysis of $G_{A}$ data. The best procedure for selecting the most appropriate parameters and then finding the optimal parametrization form is performing a parametrization scan as described in Ref. [91] for the case of PDFs determination through a QCD analysis of HERA DIS data.

First, we consider the profile function (18) and again use the reduced set of $G_{A}$ data. The value of $\mu$ in which PPDFs are chosen is set to the value obtained in Eq. (15) using the simple Ansatz of Eq. (7). By performing a parametrization scan, it is found that none of the free parameters can lead to a decrease in the value of $\chi^{2}$ more than one unit as compared to the corresponding value for the analysis using the simple Ansatz (see the previous subsection). Consequently, adding some free parameters in the form of Eq. (18), even for the valence quark profile functions, will not have any effect on the fit quality. However, if we use the profile function (19) instead, some improvements can be achieved in the fit quality. We find that the only parameters that can lead to a significant decrease in the value of $\chi^{2}$ are the valence quarks parameters. Moreover, by considering $A_{u_{v}}, B_{u_{v}}, A_{d_{v}}$, and $B_{d_{v}}$ as free parameters and setting the other parameters for $\bar{u}$ and $\bar{d}$ equal to zero, the value of $\chi^{2}$ decreases from 184.1 [which is $\chi^{2}$ for the analysis of reduced $G_{A}$ data using the simple Ansatz of Eq. (7)] to 173.6. Next, we investigate the possibility of assuming $u_{v}$ and $d_{v}$ parameters to be equal, to reduce the number of free parameter as much as possible without damaging the quality of the fit. For this purpose, we consider $A_{u_{v}}=$ $A_{d_{v}}=A_{v}$ and $B_{u_{v}}=B_{d_{v}}=B_{v}$ so that only two extra parameters contribute in the fit. The value of $\mu$ is again set to the value obtained in Eq. (15). As a result, we find that the value of $\chi^{2}$ changes less than two units, namely, from 173.6 to 175.0. This means that it is acceptable to take the $u_{v}$ and $d_{v}$ parameters to be equal and reduce the 


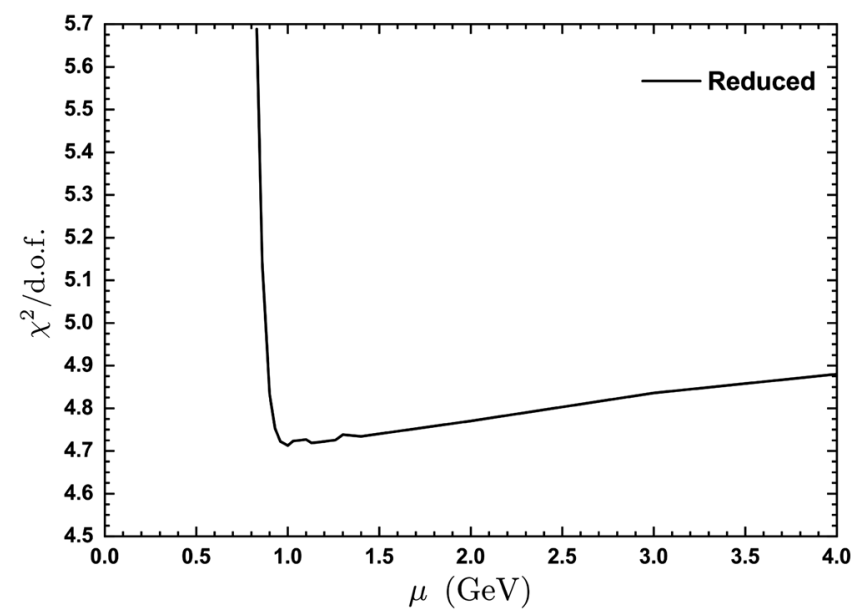

FIG. 8. Same as Fig. 4, but using Eq. (19) for the profile function.

number of free parameters. Then, the optimal values obtained for the parameters of the fit are as follows:

$$
\begin{aligned}
\alpha^{\prime} & =1.029 \pm 0.22 \mathrm{GeV}^{-2}, \\
A_{v} & =12.74 \pm 2.20 \mathrm{GeV}^{-2}, \quad B_{v}=-3.5 \pm 0.64 \mathrm{GeV}^{-2} .
\end{aligned}
$$

As can be seen, the value of $\alpha^{\prime}$ now has increased to about 1.0 , which is consistent with the result obtained in Ref. [31].

For the analyses performed so far in this subsection, we have set the value of $\mu$ in which PPDFs are chosen equal to $\mu=0.962 \mathrm{GeV}$ according to Eq. (15). However, we should find the best value of $\mu$ by performing a minimum tracing or considering it as a free parameter of the fit just like the previous section. Figure 8 shows the results obtained by minimum tracing of $\chi^{2} /$ d.o.f. values of the reduced $G_{A}$ data as a function of $\mu$ using the profile function (19) with the same $A_{v}$ and $B_{v}$ parameters for valence quarks and setting the corresponding sea quark parameters equal to zero. According to this figure, the minimum occurs at about $\mu=1.0 \mathrm{GeV}$, which is somewhat larger than before but still smaller than $\mu=2.0 \mathrm{GeV}$, which has been considered in Ref. [31]. In this situation, the optimal values obtained for the parameters of the fit are as follows:

$$
\begin{aligned}
\alpha^{\prime} & =1.054 \pm 0.22 \mathrm{GeV}^{-2}, \\
A_{v} & =13.28 \pm 2.00 \mathrm{GeV}^{-2}, \quad B_{v}=-3.64 \pm 0.64 \mathrm{GeV}^{-2} .
\end{aligned}
$$

Next, we examine the effect of considering $\mu$ as a free parameter of which the value is to be determined by simultaneous optimization along with the other three parameters. We find that the results do not change significantly, just like before. The results are
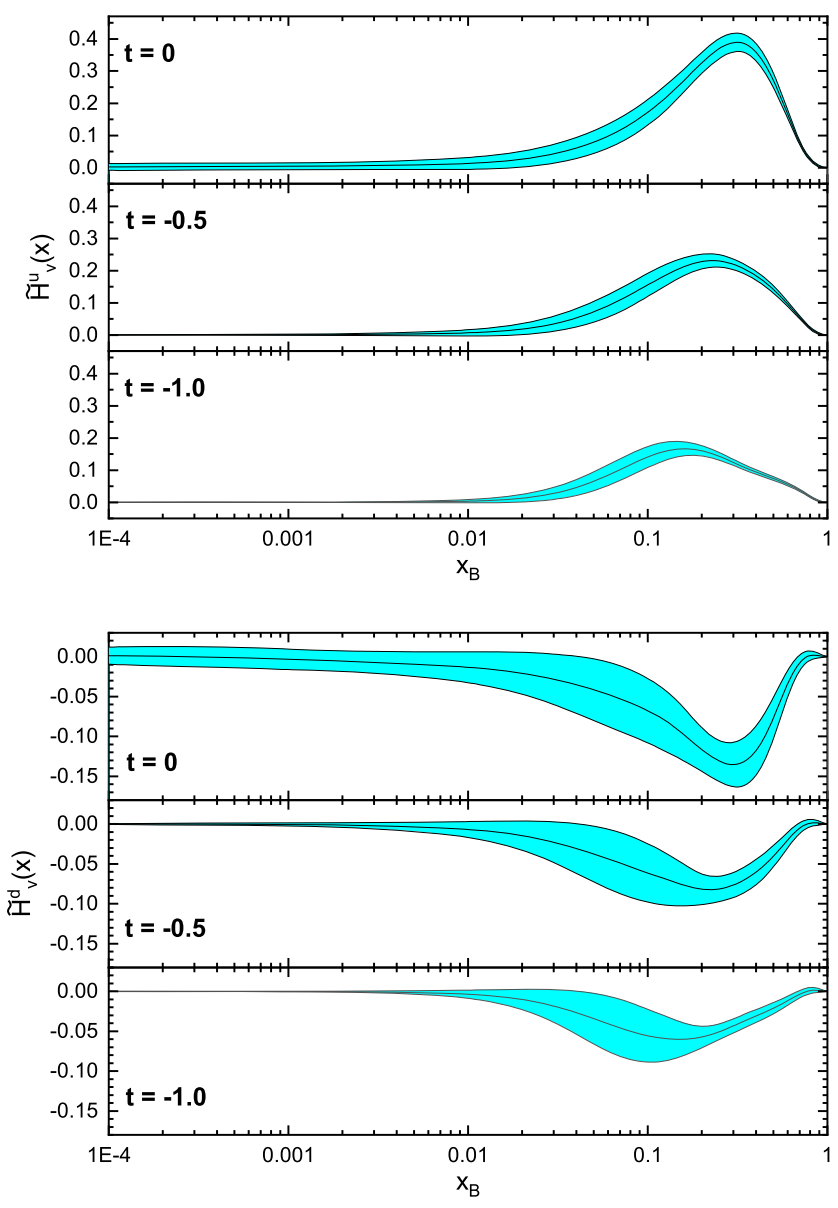

FIG. 9. The polarized GPDs $\tilde{H}_{u_{v}}$ (top) and $\tilde{H}_{d_{v}}$ (bottom) as a function of $x$ at $\mu=1 \mathrm{GeV}$ for three different values of $t=0,-0.5$, and $-1 \mathrm{GeV}^{2}$. The theoretical calculations have been performed using the Ansatz of Eq. (11) with profile function (19) and values of Eq. (22).

$$
\begin{aligned}
\alpha^{\prime} & =1.054 \pm 0.22 \mathrm{GeV}^{-2}, \quad \mu=0.997 \pm 0.363 \mathrm{GeV} \\
A_{v} & =13.28 \pm 2.00 \mathrm{GeV}^{-2}, \quad B_{v}=-3.64 \pm 0.64 \mathrm{GeV}^{-2}
\end{aligned}
$$

All things considered, we can conclude that using reduced set of $G_{A}$ data to determine the best value of $\mu$, in which PPDFs are chosen, leads to a smaller amount for it (about $\mu=1.0 \mathrm{GeV}$ ) as compared to the value assumed in Ref. [31], whether a simple Ansatz is used or a more flexible Ansatz like Eq. (19) is used. However, for the case of $\alpha^{\prime}$, the situation is somewhat different. Actually, using the simple Ansatz of Eq. (7) leads to $\alpha^{\prime}=0.59$, which is smaller than the one obtained by the study of strange Dirac form factor $F_{1}^{s}$ [31], while using a complex Ansatz like Eq. (19) leads to a value about $\alpha^{\prime}=1.054 \mathrm{GeV}^{-2}$, which is in consistent with the result of Ref. [31].

It is also of interest now to plot polarized GPDs according to the Ansatz of Eq. (11) and using profile function (19) and values shown in Eq. (22) obtained from final analysis. Figure 9 shows polarized GPDs $\tilde{H}_{u_{v}}$ (top) 

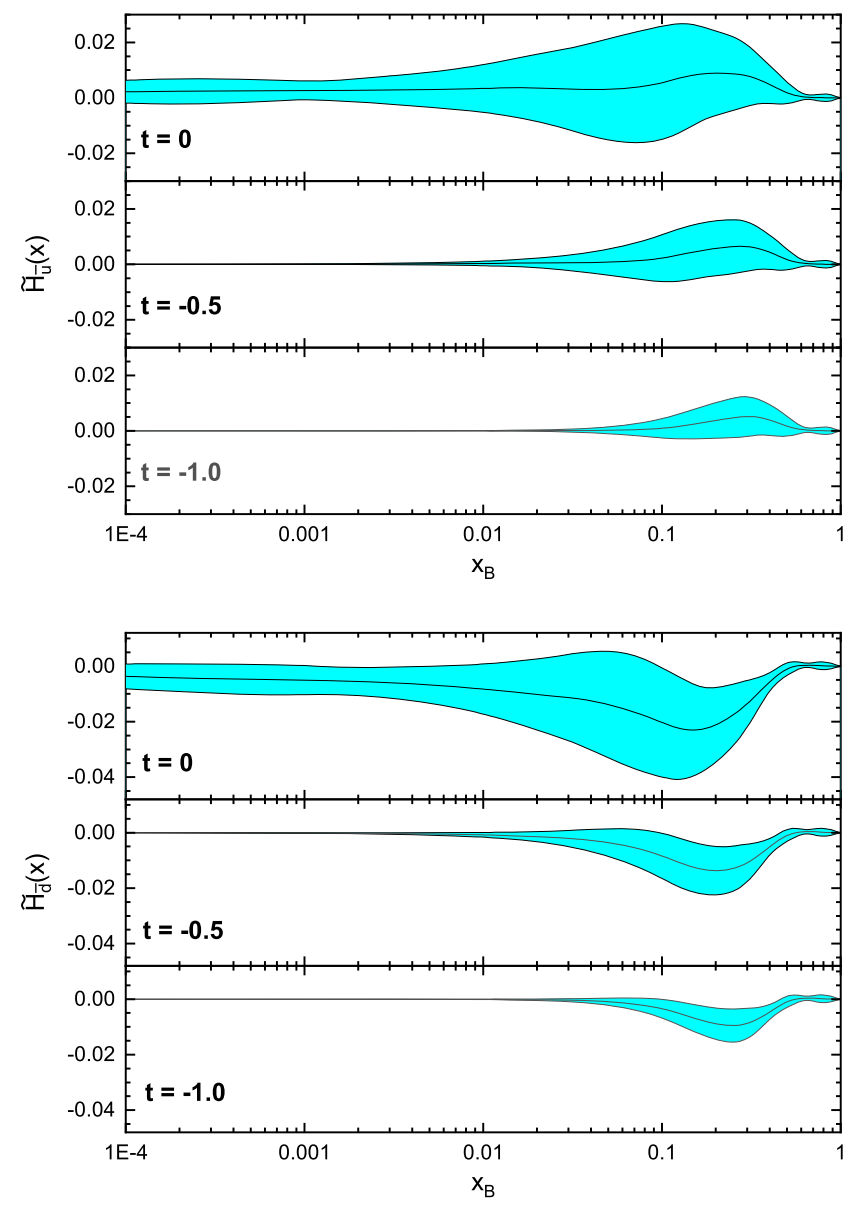

FIG. 10. Same as Fig. 9, but for $\tilde{H}_{\bar{u}}$ and $\tilde{H}_{\bar{d}}$.

and $\tilde{H}_{d_{v}}$ (bottom), as a function of $x$ for three different values of $t=0,-0.5$ and $-1 \mathrm{GeV}^{2}$. Note that for $t=0$ we obtain the original polarized PDFs from NNPDFPOL1.1 [6]. As can be seen, as the absolute value of $t$ increases, the distributions for the valence quarks decrease in magnitude and shift somewhat to smaller values of $x$, as expected. Note that the uncertainty of $\tilde{H}_{u_{v}}$ is less than $\tilde{H}_{d_{v}}$, since the $\Delta u_{v}$ PPDF of NNPDFPOL1.1 has less uncertainty. The corresponding plots for $\tilde{H}_{\bar{u}}$ and $\tilde{H}_{\bar{d}}$ are shown in Fig. 10 . Note that in this case the parameters $A$ and $B$ in profile function (19) are equal to zero. This figure shows that as the absolute value of $t$ increases the distributions for the sea quarks slightly decrease in magnitude and shift to larger $x$. However, their uncertainty bands are greater than those of the valence quarks.

We can now compare the result of our final analysis to the experimental data. Figure 11 shows a comparison between the theoretical predictions for the nucleon $\mathrm{AFF} G_{A}$ obtained using profile function (19) with values from Eq. (21) (filled band) and reduced $G_{A}$ data. Note that NNPDFPOL1.1 PPDFs have been chosen at $\mu=0.997 \mathrm{GeV}$ as shown in Eq. (22). As can be seen, the theoretical prediction is in a good agreement with the experimental data.

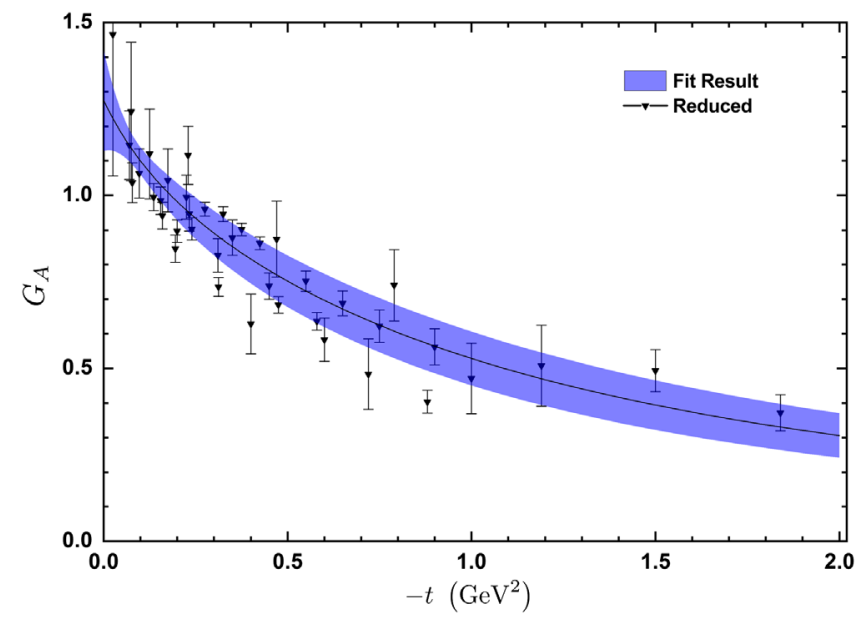

FIG. 11. A comparison between the reduced experimental data and theoretical predictions for $G_{A}$ obtained using profile function (19) with values of parameters given in Eq. (22) (filled band). NNPDFPOL1.1 PPDFs have been chosen at $\mu=0.997 \mathrm{GeV}$.

In addition to the AFF data that can be used to constrain polarized GPDs at smaller values of $-t$, some information about another moment of $\tilde{H}$ can be obtained from wideangle Compton scattering data [92], which also constrain the large $-t$ behavior of $\tilde{H}$. In this way, it is also of interest to compare the final results obtained from our analysis to those of, for example, Ref. [92]. We compared these two GPD sets and found that there are considerable differences between them. Therefore, it would be interesting, as a separate investigation, to analyze both the AFFs and wideangle Compton scattering data simultaneously to determined polarized GPDs that are compatible with both sets of experimental data and cover a wider range of $-t$.

\section{SUMMARY AND CONCLUSIONS}

An accurate knowledge of generalized parton distributions is necessary for describing hard exclusive electroproduction processes. GPDs are nonperturbative objects, which can be determined using analysis of experimental data from exclusive processes such as DVCS and meson production. One of the important properties of GPDs is their mutual relations with PDFs and form factors. To be more precise, (polarized) GPDs reduce to (polarized) PDFs in the limit of zero momentum transfer. On the other hand, the integration of GPDs over Bjorken $x$ yields Dirac and Pauli form factors. This procedure for the case of polarized GPDs yields AFFs or intrinsic quark contribution to the nucleon spin. In the present work, considering the DFJK model [30-32] to relate GPDs and PDFs, we have calculated polarized GPDs $\left(\tilde{H}_{q}\right)$ using predetermined polarized PDFs and have studied in detail the axial form factor of nucleon $G_{A}$. As a result, we have shown that our model to calculate $G_{A}$ is not sensitive to the choice of PPDFs set, such that the difference between the results 
obtained using the DSSV08 and NNPDFPOL1.1 PPDFs is almost less than $2 \%$ in the full range of $-t$. By studying the dependence of $G_{A}$ on the value of scale $\mu$ at which PPDFs are chosen, we can also find that as $\mu$ decreases $G_{A}$ increases, especially for larger values of $-t$, so the difference between the results of $\mu=1$ and $\mu=2 \mathrm{GeV}$ reaches $30 \%$ at $-t=2 \mathrm{GeV}^{2}$. Overall, we have concluded that taking PPDFs at a lower scale $\mu$ can lead to a better description of the experimental data. Moreover, we have investigated the model uncertainties imposed on $G_{A}$ due to the PPDFs uncertainties and also variation of $\alpha^{\prime}$ in profile functions $f(x)$. We have indicated that the uncertainty arising from the $\alpha^{\prime}$ variations is dominant as compared to the PPDFs uncertainty, except for very small values of $-t$ in which the PPDFs uncertainty becomes dominant. Moreover, by considering different scenarios, we have determined the optimal values of parameters of the model using standard $\chi^{2}$ analysis of the available experimental data related to nucleon axial form factor. We used both a simple and complex profile function to find the best conditions for obtaining better consistency between the theoretical predictions and experimental data. We have shown that using $G_{A}$ data to determine the best value of $\mu$, in which
PPDFs are chosen, leads to a smaller amount for it (about $\mu=1.0 \mathrm{GeV}$ ) compared with the value assumed in Ref. [31], whether one uses a simple Ansatz or a more flexible Ansatz. In addition, using a simple Ansatz leads to a smaller value for $\alpha^{\prime}$ rather than one obtained by the study of strange Dirac form factor $F_{1}^{s}$ [31], while using a complex Ansatz leads to $\alpha^{\prime}=1.054 \mathrm{GeV}^{-2}$, which is consistent with the result of Ref. [31]. More precise measurements of the neutrino cross section on hydrogen and deuterium are needed to unravel the axial structure of the nucleon.

\section{ACKNOWLEDGMENTS}

We thank H. Abedi and M. J. Kazemi for reading the manuscript and for stimulating discussions. We also wish to express our gratitude toward U. G. Meissner and A. Butkevich for providing us with the data. H. H. and S.S. G. would like to thank the research council of the Shahid Beheshti University for financial support. M. G. thanks the School of Particles and Accelerators, Institute for Research in Fundamental Sciences (IPM), for financial support provided for this research.
[1] J. Ashman et al. (European Muon Collaboration), A measurement of the spin asymmetry and determination of the structure function $\mathrm{g}(1)$ in deep inelastic Muon-Proton scattering, Phys. Lett. B 206, 364 (1988).

[2] D. de Florian, R. Sassot, M. Stratmann, and W. Vogelsang, Extraction of spin-dependent parton densities and their uncertainties, Phys. Rev. D 80, 034030 (2009).

[3] J. Blumlein and H. Bottcher, QCD analysis of polarized deep inelastic scattering data, Nucl. Phys. B841, 205 (2010).

[4] E. Leader, A. V. Sidorov, and D. B. Stamenov, Determination of polarized PDFs from a QCD analysis of inclusive and semi-inclusive deep inelastic scattering data, Phys. Rev. D 82, 114018 (2010).

[5] D. de Florian, R. Sassot, M. Stratmann, and W. Vogelsang, Evidence for Polarization of Gluons in the Proton, Phys. Rev. Lett. 113, 012001 (2014).

[6] E. R. Nocera, R. D. Ball, S. Forte, G. Ridolfi, and J. Rojo (NNPDF Collaboration), A first unbiased global determination of polarized PDFs and their uncertainties, Nucl. Phys. B887, 276 (2014).

[7] P. Jimenez-Delgado, H. Avakian, and W. Melnitchouk (JAM Collaboration), Constraints on spin-dependent parton distributions at large $\mathrm{x}$ from global QCD analysis, Phys. Lett. B 738, 263 (2014).

[8] N. Sato, W. Melnitchouk, S. E. Kuhn, J. J. Ethier, and A. Accardi (JAM Collaboration), Iterative Monte Carlo analysis of spin-dependent parton distributions, Phys. Rev. D 93, 074005 (2016).
[9] F. Taghavi-Shahri, H. Khanpour, S. Atashbar Tehrani, and Z. Alizadeh Yazdi, Next-to-next-to-leading order QCD analysis of spin-dependent parton distribution functions and their uncertainties: Jacobi polynomials approach, Phys. Rev. D 93, 114024 (2016).

[10] H. Khanpour, S. T. Monfared, and S. Atashbar Tehrani, Nucleon spin structure functions at NNLO in the presence of target mass corrections and higher twist effects, Phys. Rev. D 95, 074006 (2017).

[11] J. J. Ethier, N. Sato, and W. Melnitchouk, First Simultaneous Extraction of Spin-Dependent Parton Distributions and Fragmentation Functions from a Global QCD Analysis, Phys. Rev. Lett. 119, 132001 (2017).

[12] H. Khanpour, S. T. Monfared, and S. Atashbar Tehrani, Study of spin-dependent structure functions of ${ }^{3} \mathrm{He}$ and ${ }^{3} \mathrm{H}$ at NNLO approximation and corresponding nuclear corrections, Phys. Rev. D 96, 074037 (2017).

[13] M. Salajegheh, S. M. Moosavi Nejad, H. Khanpour, and S. Atashbar Tehrani, Analytical approaches to the determination of spin-dependent parton distribution functions at NNLO approximation, Phys. Rev. C 97, 055201 (2018).

[14] K. Goeke, M. V. Polyakov, and M. Vanderhaeghen, Hard exclusive reactions and the structure of hadrons, Prog. Part. Nucl. Phys. 47, 401 (2001).

[15] M. Diehl, Generalized parton distributions, Phys. Rep. 388, 41 (2003). 
[16] M. V. Polyakov, Generalized parton distributions and strong forces inside nucleons and nuclei, Phys. Lett. B 555, 57 (2003).

[17] A. Freund, M. McDermott, and M. Strikman, Modeling generalized parton distributions to describe deeply virtual Compton scattering data, Phys. Rev. D 67, 036001 (2003).

[18] S. Scopetta and V. Vento, Generalized parton distributions and composite constituent quarks, Phys. Rev. D 69, 094004 (2004).

[19] A. V. Belitsky and A. V. Radyushkin, Unraveling hadron structure with generalized parton distributions, Phys. Rep. 418, 1 (2005).

[20] S. Boffi and B. Pasquini, Generalized parton distributions and the structure of the nucleon, Riv. Nuovo Cimento 30, 387 (2007).

[21] V. Guzey and M. V. Polyakov, Dual parameterization of generalized parton distributions and description of DVCS data, Eur. Phys. J. C 46, 151 (2006).

[22] V. Guzey and T. Teckentrup, The dual parameterization of the proton generalized parton distribution functions $\mathrm{H}$ and $\mathrm{E}$ and description of the DVCS cross sections and asymmetries, Phys. Rev. D 74, 054027 (2006).

[23] P. Hagler et al. (LHPC Collaboration), Nucleon generalized parton distributions from full lattice QCD, Phys. Rev. D 77, 094502 (2008).

[24] C. Alexandrou, M. Constantinou, S. Dinter, V. Drach, K. Jansen, C. Kallidonis, and G. Koutsou, Nucleon form factors and moments of generalized parton distributions using $N_{f}=2+1+1$ twisted mass fermions, Phys. Rev. D 88, 014509 (2013).

[25] K. Kumericki, D. Mueller, and K. Passek-Kumericki, Towards a fitting procedure for deeply virtual Compton scattering at next-to-leading order and beyond, Nucl. Phys. B794, 244 (2008).

[26] M. Guidal, H. Moutarde, and M. Vanderhaeghen, Generalized parton distributions in the valence region from deeply virtual Compton scattering, Rep. Prog. Phys. 76, 066202 (2013).

[27] K. Kumericki, S. Liuti, and H. Moutarde, GPD phenomenology and DVCS fitting: Entering the high-precision era, Eur. Phys. J. A 52, 157 (2016).

[28] H. Khanpour, M. Goharipour, and V. Guzey, Effects of nextto-leading order DGLAP evolution on generalized parton distributions of the proton and deeply virtual Compton scattering at high energy, Eur. Phys. J. C 78, 7 (2018).

[29] D. Y. Ivanov, A. Schafer, L. Szymanowski, and G. Krasnikov, Exclusive photoproduction of a heavy vector meson in QCD, Eur. Phys. J. C 34, 297 (2004); 75, 75(E) (2015).

[30] M. Diehl, T. Feldmann, R. Jakob, and P. Kroll, Generalized parton distributions from nucleon form-factor data, Eur. Phys. J. C 39, 1 (2005).

[31] M. Diehl, T. Feldmann, and P. Kroll, Form factors and other measures of strangeness in the nucleon, Phys. Rev. D 77, 033006 (2008).

[32] M. Diehl and P. Kroll, Nucleon form factors, generalized parton distributions and quark angular momentum, Eur. Phys. J. C 73, 2397 (2013).

[33] M. Burkardt, Impact parameter dependent parton distributions and transverse single spin asymmetries, Phys. Rev. D 66, 114005 (2002).
[34] N. d'Hose, E. Burtin, P. A. M. Guichon, and J. Marroncle, Feasibility study of deeply virtual Compton scattering using COMPASS at CERN, Eur. Phys. J. A 19, 47 (2004).

[35] W. Armstrong et al., Partonic structure of light nuclei, arXiv:1708.00888.

[36] A. Accardi et al., Electron ion collider: The next QCD frontier: Understanding the glue that binds us all, Eur. Phys. J. A 52, 268 (2016).

[37] K. Tsushima, T. Yamaguchi, Y. Kohyama, and K. Kubodera, Weak interaction form-factors and magnetic moments of octet baryons: Chiral bag model with Gluonic effects, Nucl. Phys. A489, 557 (1988).

[38] B. Julia-Diaz, D. O. Riska, and F. Coester, Axial transition form-factors and pion decay of baryon resonances, Phys. Rev. C 70, 045204 (2004).

[39] S. Mamedov, B. B. Sirvanli, I. Atayev, and N. Huseynova, Nucleon's axial-vector form factor in the hard-wall AdS/ QCD model, Int. J. Theor. Phys. 56, 1861 (2017).

[40] X. Y. Liu, K. Khosonthongkee, A. Limphirat, and Y. Yan, Comparisons of magnetic charge and axial charge meson cloud distributions in the PCQM, Sci. Rep. 7, 8139 (2017).

[41] I. V. Anikin, V. M. Braun, and N. Offen, Axial form factor of the nucleon at large momentum transfers, Phys. Rev. D 94, 034011 (2016).

[42] C. Adamuscin, E. Tomasi-Gustafsson, E. Santopinto, and R. Bijker, Two-component model for the axial form factor of the nucleon, Phys. Rev. C 78, 035201 (2008).

[43] I. G. Aznauryan et al., Studies of nucleon resonance structure in exclusive meson electroproduction, Int. J. Mod. Phys. E 22, 1330015 (2013).

[44] G. Ramalho, Holographic estimate of the meson cloud contribution to nucleon axial form factor, Phys. Rev. D 97, 073002 (2018).

[45] V. Bernard, L. Elouadrhiri, and U. G. Meissner, Axial structure of the nucleon: Topical review, J. Phys. G 28, R1 (2002).

[46] M. R. Schindler and S. Scherer, Nucleon form factors of the isovector axial-vector current: Situation of experiments and theory, Eur. Phys. J. A 32, 429 (2007).

[47] T. Bhattacharya, S. D. Cohen, R. Gupta, A. Joseph, H. W. Lin, and B. Yoon, Nucleon charges and electromagnetic form factors from $2+1+1$-flavor lattice QCD, Phys. Rev. D 89, 094502 (2014).

[48] J. Liang, Y. B. Yang, K. F. Liu, A. Alexandru, T. Draper, and R. S. Sufian, Lattice calculation of nucleon isovector axial charge with improved currents, Phys. Rev. D 96, 034519 (2017).

[49] J. Green, N. Hasan, S. Meinel, M. Engelhardt, S. Krieg, J. Laeuchli, J. Negele, K. Orginos, A. Pochinsky, and S. Syritsyn, Up, down, and strange nucleon axial form factors from lattice QCD, Phys. Rev. D 95, 114502 (2017).

[50] D. L. Yao, L. Alvarez-Ruso, and M. J. Vicente-Vacas, Extraction of nucleon axial charge and radius from lattice QCD results using baryon chiral perturbation theory, Phys. Rev. D 96, 116022 (2017).

[51] A. Abdel-Rehim et al., Nucleon and pion structure with lattice QCD simulations at physical value of the pion mass, Phys. Rev. D 92, 114513 (2015); 93, 039904(E) (2016).

[52] G. S. Bali, S. Collins, B. Gläßle, M. Göckeler, J. Najjar, R. H. Rödl, A. Schäfer, R.W. Schiel, W. Söldner, and 
A. Sternbeck, Nucleon isovector couplings from $N_{f}=2$ lattice QCD, Phys. Rev. D 91, 054501 (2015).

[53] T. Bhattacharya, V. Cirigliano, S. Cohen, R. Gupta, H. W. Lin, and B. Yoon, Axial, scalar and tensor charges of the nucleon from $2+1+1$-flavor lattice QCD, Phys. Rev. D 94, 054508 (2016).

[54] K. I. Ishikawa, Y. Kuramashi, S. Sasaki, N. Tsukamoto, A. Ukawa, and T. Yamazaki (PACS Collaboration), Nucleon form factors on a large volume lattice near the physical point in $2+1$ flavor QCD, Phys. Rev. D 98, 074510 (2018).

[55] L. Alvarez-Ruso, K. M. Graczyk, and E. Saul-Sala, Nucleon axial form factor from a Bayesian neural-network analysis of neutrino-scattering data, Phys. Rev. C 99, 025204 (2019).

[56] B. Pasquini, M. Pincetti, and S. Boffi, Chiral-odd generalized parton distributions in constituent quark models, Phys. Rev. D 72, 094029 (2005).

[57] B. Pasquini and S. Boffi, Virtual meson cloud of the nucleon and generalized parton distributions, Phys. Rev. D 73, 094001 (2006).

[58] H. Dahiya and A. Mukherjee, Chiral odd generalized parton distributions in impact parameter space, Phys. Rev. D 77, 045032 (2008).

[59] T. Frederico, E. Pace, B. Pasquini, and G. Salme, Pion generalized parton distributions with covariant and lightfront constituent quark models, Phys. Rev. D 80, 054021 (2009).

[60] A. Mukherjee, S. Nair, and V. Kumar Ojha, Generalized parton distributions of the photon with helicity flip, Phys. Lett. B 721, 284 (2013).

[61] T. Maji, C. Mondal, D. Chakrabarti, and O. V. Teryaev, Relating transverse structure of various parton distributions, J. High Energy Phys. 01 (2016) 165.

[62] G. R. Goldstein, J. O. Hernandez, and S. Liuti, Flexible parametrization of generalized parton distributions from deeply virtual compton scattering observables, Phys. Rev. D 84, 034007 (2011).

[63] G. R. Goldstein, J. O. Gonzalez Hernandez, and S. Liuti, Flexible parametrization of generalized parton distributions: The chiral-odd sector, Phys. Rev. D 91, 114013 (2015).

[64] N. Sharma, Momentum transfer dependence of generalized parton distributions, Eur. Phys. J. A 52, 338 (2016).

[65] J. C. Collins and A. Freund, Proof of factorization for deeply virtual Compton scattering in QCD, Phys. Rev. D 59, 074009 (1999).

[66] J. C. Collins, L. Frankfurt, and M. Strikman, Factorization for hard exclusive electroproduction of mesons in QCD, Phys. Rev. D 56, 2982 (1997).

[67] X. D. Ji, Gauge-Invariant Decomposition of Nucleon Spin, Phys. Rev. Lett. 78, 610 (1997).

[68] A. V. Butkevich and D. Perevalov, Determination of the axial nucleon form factor from the MiniBooNE data, Phys. Rev. D 89, 053014 (2014).

[69] E. Amaldi, B. Borgia, P. Pistilli, M. Balla, G. V. Di Giorgio, A. Giazotto, S. Serbassi, and G. Stoppini, On pion electroproduction at $5 \mathrm{fm}$-squared near threshold, Nuovo Cimento A 65, 377 (1970).

[70] E. Amaldi, M. Benevantano, B. Borgia, F. De Notaristefani, A. Frondaroli, P. Pistilli, I. Sestili, and M. Severi,
Axial-vector form-factor of the nucleon from a coincidence experiment on electroproduction at threshold, Phys. Lett. 41B, 216 (1972).

[71] E. D. Bloom, R. L. A. Cottrell, H. DeStaebler, C. L. Jordan, H. G. Piel, C. Y. Prescott, R. Siemann, S. Stein, and R. E. Taylor, Measurements Of Inelastic Electron Scattering Cross-sections Near One Pion Threshold, Phys. Rev. Lett. 30, 1186 (1973).

[72] P. Brauel et al., $\pi^{+}$electroproduction on hydrogen near threshold at four-momentum transfers of $0.2,0.4$ and $0.6 \mathrm{GeV}^{2}$, Phys. Lett. 45B, 389 (1973).

[73] A. Del Guerra, A. Giazotto, M. A. Giorgi, A. Stefanini, D. R. Botterill, D. W. Braben, D. Clarke, and P. R. Norton, Measurements of threshold pi + electroproduction at low momentum transfer, Nucl. Phys. B99, 253 (1975).

[74] A. Del Guerra, A. Giazotto, M. A. Giorgi, A. Stefanini, D. R. Botterill, H. E. Montgomery, P. R. Norton, and G. Matone, Threshold $\pi^{+}$electroproduction at high momentum transfer: A determination of the nucleon axial vector formfactor, Nucl. Phys. B107, 65 (1976).

[75] P. Joos et al., Determination of the nucleon axial vector form-factor from pi delta electroproduction near threshold, Phys. Lett. 62B, 230 (1976).

[76] A. S. Esaulov, A. M. Pilipenko, and Y. I. Titov, Longitudinal and transverse contributions to the threshold cross-section slope of single pion electroproduction by a proton, Nucl. Phys. B136, 511 (1978).

[77] S. Choi et al., Axial and Pseudoscalar Nucleon FormFactors from Low-Energy Pion Electroproduction, Phys. Rev. Lett. 71, 3927 (1993).

[78] S. Choi, Etude experimentale et theorique de lelectroproduction de pions pres du seuil mesure des facteurs de forme axial et pseudo-scalaire, Thesis No. 2643, Universite Paris XI Orsay, 1993.https://www.theses.fr/ 1993PA112135

[79] Y. Nambu and D. Lurie, Chirality conservation and soft pion production, Phys. Rev. 125, 1429 (1962).

[80] Y. Nambu and E. Shrauner, Soft pion emission induced by electromagnetic and weak interactions, Phys. Rev. 128, 862 (1962).

[81] B. Bhattacharya, R. J. Hill, and G. Paz, Model independent determination of the axial mass parameter in quasielastic neutrino-nucleon scattering, Phys. Rev. D 84, 073006 (2011).

[82] B. Bhattacharya, G. Paz, and A. J. Tropiano, Modelindependent determination of the axial mass parameter in quasielastic antineutrino-nucleon scattering, Phys. Rev. D 92, 113011 (2015).

[83] A. S. Meyer, M. Betancourt, R. Gran, and R. J. Hill, Deuterium target data for precision neutrino-nucleus cross sections, Phys. Rev. D 93, 113015 (2016).

[84] M. Gonzalez-Alonso, O. Naviliat-Cuncic, and N. Severijns, New physics searches in nuclear and neutron $\beta$ decay, Prog. Part. Nucl. Phys. 104, 165 (2019).

[85] M. Tanabashi et al., Review of particle physics, Phys. Rev. D 98, 030001 (2018).

[86] A. Buckley, J. Ferrando, S. Lloyd, K. Nordström, B. Page, M. Rüfenacht, M. Schönherr, and G. Watt, LHAPDF6: Parton density access in the LHC precision era, Eur. Phys. J. C 75, 132 (2015). 
[87] J. Pumplin, D. Stump, R. Brock, D. Casey, J. Huston, J. Kalk, H. L. Lai, and W. K. Tung, Uncertainties of predictions from parton distribution functions. 2. The Hessian method, Phys. Rev. D 65, 014013 (2001).

[88] P. M. Nadolsky, H. L. Lai, Q. H. Cao, J. Huston, J. Pumplin, D. Stump, W. K. Tung, and C.-P. Yuan, Implications of CTEQ global analysis for collider observables, Phys. Rev. D 78, 013004 (2008).

[89] F. James and M. Roos, Minuit: A system for function minimization and analysis of the parameter errors and correlations, Comput. Phys. Commun. 10, 343 (1975).
[90] S. V. Goloskokov and P. Kroll, Transversity in hard exclusive electroproduction of pseudoscalar mesons, Eur. Phys. J. A 47, 112 (2011).

[91] F. D. Aaron et al. (H1 and ZEUS Collaborations), Combined measurement and QCD analysis of the inclusive $e+-$ p scattering cross sections at HERA, J. High Energy Phys. 01 (2010) 109.

[92] P. Kroll, The GPD $\tilde{H}$ and spin correlations in wide-angle Compton scattering, Eur. Phys. J. A 53, 130 (2017). 